\title{
La forme verbale " être + participe passé » en tant que marqueur d'aspect et de structure argumentale : une typologie graduée
}

\author{
Anne Buchard \\ Université de Valenciennes \\ anne.buchard@free.fr \\ Anne Carlier \\ Savoirs, Textes, Langage (STL) \\ anne.carlier@univ-valenciennes.fr
}

Rares sont les études qui ont tenté d'assigner à la forme verbale combinant l'auxiliaire être avec le participe passé une signification grammaticale unitaire. Comme l'a montré Creissels (2002), cette forme verbale peut se rattacher à des constructions verbales diverses :
(1) Pierre est monté.
(2) Pierre est divorcé.
(3) Le sucre est caramélisé.
(4) Le verre est renversé.
(5) Marie est couchée dans le canapé.

Les séquences «être + participe passé » dans (1) et (2), dont le verbe est intransitif, ont été abordées en termes de temps et / ou d'aspect, alors que celles dans (4) et (5), formées à partir d'un verbe susceptible d'un emploi transitif et analysées respectivement comme un passif et une structure liée à la construction pronominale, ont surtout été étudiées en termes de structure argumentale. Quant à «être + participe passé » dans (3), dont le verbe présente une construction transitive et une construction intransitive, cette forme verbale est considérée dans cette optique comme ayant une signification hybride, ayant trait tant à l'aspect qu'à la structure argumentale. Un second clivage introduit dans les études consacrées plus spécifiquement aux valeurs aspectuelles des différentes instances de la forme "être + participe passé » concerne le statut catégoriel du participe passé, verbal ou adjectival : alors que le participe passé est clairement verbal dans le cas du passé composé en (1), il serait adjectival dans (2), (3) et (5) et pourrait être adjectival ou verbal dans le cas du passif en (4).

La présente communication se propose de montrer que, dans tous les cas, être est à la fois un marqueur d'aspect et un marqueur de structure argumentale $(\S 1)$. Elle fera ensuite ressortir que les différentes instances de la forme "être + participe passé » forment un continuum allant d'une structure attributive adjectivale à une structure verbale $(\S 2)$. L'analyse synchronique sera enfin appuyée à partir de la diachronie $(\S 3)$ : il sera montré comment la séquence «être + participe passé », à l'origine adjectivale, s'est intégrée progressivement dans la flexion verbale pour devenir conjointement un marqueur de structure argumentale et d'aspect. 


\section{La forme « être + participe passé » : marqueur d'aspect et de structure argumentale}

\subsection{Les verbes intransitifs ayant un passé composé en "être + participe passé $»$}

\subsection{1 "être + participe passé » : un marqueur du temps et de l'aspect}

L'ensemble des travaux linguistiques consacrés aux temps verbaux s'accordent à dire que, comme toutes les formes verbales composées, le passé composé présente au moins deux valeurs : une valeur de présent d'aspect accompli et une valeur d'antériorité par rapport au moment présent (e.a. Damourette et Pichon 1940-1944 ; Wilmet 1970, 1992 ; Martin 1971 ; Vet 1992 ; Gosselin 1996 ; Luscher et Sthioul 1996).

- Dans un contexte présent, le passé composé tend à être interprété comme un présent d'aspect accompli.

(6) Je vois que Pierre est sorti. (Vet, 1992:46)

La plupart des verbes intransitifs sélectionnant l'auxiliaire être sont des verbes téliques ${ }^{1}$ : ils marquent à l'aspect accompli la valeur d'état résultant ainsi qu'en témoigne leur compatibilité avec le complément aspectuel de durée introduit par depuis.

(7) Elle est arrivée depuis longtemps? (A. Robbe-Grillet, Les Gommes)

Certains verbes intransitifs sélectionnant l'auxiliaire être, bien que téliques, ne conduisent pas à un état résultant : ils marquent une incidence sur le moment présent.

(8) Je reste, puisqu'elle est venue (*depuis un quart d'heure).

- En présence de formes verbales passées et/ou de compléments temporels se rapportant au passé, le passé composé prend la valeur d'antériorité par rapport au moment de l'énonciation.

(9) Paul est parti à huit heures.

La forme verbale évoque alors le procès dans son déroulement, lequel peut être caractérisé par des adverbes :

(10) L'autre soir, avant l'orage, il est sorti lentement de sous les branches basses une religieuse et une jeune fille. (Alain-Fournier, Correspondance avec J. Rivière)

La valeur d'antériorité par rapport au présent est dérivée par rapport à celle du présent d'aspect accompli (Cohen 1989). Le passage de l'une à l'autre est une évolution typologiquement très répandue (Comrie 1972). Dans le contexte des langues romanes, le français représente un stade avancé de cette évolution (Harris 1987).

\subsection{2 "être + participe passé » : un marqueur de structure argumentale?}

L'ensemble des verbes se conjuguant avec être peut également être appréhendé en termes de structure argumentale dans la mesure où ces verbes sont généralement considérés comme prototypiques de la classe des inaccusatifs ( $C f$. e.a. Burzio 1986 ; Ruwet 1988 ; Herslund 1990 ; Tasmowski 1993 ; Carlier 2005 ; Lagae 2005). L'hypothèse inaccusative ${ }^{2}$ établit une distinction entre deux classes de verbes intransitifs : les inaccusatifs dont l'unique argument est un argument interne direct (11a) et les inergatifs dont l'unique argument est externe au groupe verbal (11b). 


$$
\begin{array}{ll}
\text { a. }[\mathrm{p} \varnothing[\mathrm{sv} \mathrm{V} \mathrm{SN}]] & \text { verbes inaccusatifs : aller, arriver, descendre, entrer, etc. } \\
\text { b. }\left[\mathrm{P} \mathrm{SN}\left[{ }_{\mathrm{sv}} \mathrm{V}\right]\right] & \text { verbes inergatifs : chanter, marcher, travailler, etc. }
\end{array}
$$

Cette distinction syntaxique a des corrélats sémantiques. La propriété des verbes inaccusatifs la plus souvent mise en avant dans la littérature concerne les rôles thématiques : le sujet des verbes inergatifs serait un agent, c'est-à-dire l'entité qui effectue une activité ou qui cause un changement d'état, alors que le sujet des verbes inaccusatifs correspondrait à un patient ou dans une perspective plus large à un thème, c'est-à-dire à l'entité se trouvant dans un certain état ou affectée par l'action du verbe (subissant un changement d'état ou de lieu) (Van Valin 1990). Les verbes formant leur accompli avec être présentent donc la structure argumentale suivante :

\section{Pierre est parti. Verbes sélectionnant être}

Thème

La seconde propriété sémantique des verbes inaccusatifs que mentionnent les travaux sur le sujet a trait à l'aspect lexical. Pour Tenny (1994), l'inaccusativité va de pair avec la télicité car seuls les arguments internes peuvent délimiter le procès décrit par le verbe. Les prédicats inaccusatifs, ayant pour sujet un argument interne sont donc enclins à la télicité alors que les prédicats inergatifs ayant un argument externe ne pouvant pas délimiter le procès, sont le plus souvent non téliques (R. Van Valin 1990).

Il est clair que ces deux propriétés sémantiques ne suffisent pas à rendre compte de l'ensemble de la sousclasse des verbes intransitifs auxiliés par être (Legendre et Sorace 2004). Ainsi le sujet du verbe intervenir correspond à un agent (Ruwet 1988) et rester marque l'aspect non télique (Legendre et Sorace 2004). Une troisième propriété sémantique des verbes inaccusatifs citée dans la littérature se rapporte à leur interprétation l'existentielle (Hoekstra \& Mulder 1990 ; Van de Velde 1996). Certains de ces verbes marquent en effet la venue à l'existence (advenir, apparaître, éclore, naître, survenir) ou la fin de l'existence (décéder, échoir, mourir). D'autres verbes dénotent une localisation sur le mode statique (rester) ou sur le mode dynamique en marquant un déplacement vers certain lieu comme arriver, entrer, intervenir ${ }^{3}$, monter, parvenir, venir, à partir d'un certain lieu comme descendre, partir, sortir, tomber ou encore un déplacement se caractérisant par le passage dans un certain lieu comme passer, mais ces mêmes verbes peuvent également servir de prédicat d'existence dans la mesure où 'être dans un lieu' revient à 'exister' (Carlier 2005).

L'inaccusativité ne se laisse donc pas définir par une propriété sémantique unique mais correspond à un ensemble de traits étroitement corrélés ( $c f$. Legendre \& Sorace 2004). Par ailleurs, l'existence du passé composé en "être + participe passé » est un critère suffisant, mais non nécessaire d'inaccusativité en français : certains verbes intransitifs de type inaccusatif sélectionnent l'auxiliaire avoir.

\subsection{Les verbes intransitifs dits « à double auxiliation »}

\subsection{1 "être + participe passé » : un marqueur d'aspect}

Comme l'a constaté Creissels (2000), certains passés composés à valeur résultative en «avoir + participe passé » peuvent être mis en correspondance avec des formes verbales faisant intervenir l'auxiliaire être :

(13) a. Il a divorcé.

b. [...] personne ne soupçonne qu'il a été marié une première fois et qu'il est divorcé! (P. Bourget, Lazarine)

La séquence en être sous (13b), correspondant à l'état résultant de l'événement exprimé au passé composé sous (13a), a pour origine le verbe exclusivement intransitif divorcer. Lagae (2005) relève cette même propriété pour les verbes aboutir, atterrir, disparaître, divorcer, expirer, paraître. Le fait que 
certains verbes intransitifs se conjuguant avec avoir permettent l'expression de l'état résultant au moyen de "être + participe passé ", à côté de la forme résultative en avoir, ne peut toutefois pas être réduit au phénomène lexical de "double auxiliation». Comme il ressort de notre corpus, la liste de verbes permettant la structure faisant intervenir être est loin de se limiter aux cas relevés par les grammaires :

(14) Avec la cessation de l'activité professionnelle, le travail de mémoire de la société française, les procès Touvier, Papon et d'autres, tout est resurgi de ce passé mal enfoui. (Plaidoirie de Me Rouquette, le 16 mai 2006, Procès Lipietz et consorts c/SNCF et l'Etat)

(15) Les terrains du Massif Central sont émergés dès le Permien à l'exception d'une période d'immersion allant du Lias au Jurassique. (http://www2.aclyon.fr)

(16) [...] de même que les points qui sont surgis au cours de la discussion et qui n'apparaissaient pas. dans les rapports détaillés des arbitres (www.vrr.ulaval.ca)

(17) Comme sur un bateau ceux qui jouent au bridge et lisent les revues ne conçoivent même pas le naufrage. Mais, dès que le bateau penche, ils sont naufragés [...] (Alain, Propos)

(18) [...] son corps baigne dans son sang et sa cervelle est explosée en milles morceaux autour d'elle... (forums.france $3 . \mathrm{fr}$ )

(19) Une seule céréale crue - sauf si elle est germée - est déjà très indigeste. A plus forte raison si on en mange plusieurs au même repas. (www.bien-etre.ch)

Nos matériaux vont donc à l'encontre d'une analyse purement lexicale du phénomène et constituent plutôt un argument en faveur d'un processus régulier.

\subsection{2 "être + participe passé » : un marqueur de structure argumentale?}

Après avoir soumis les verbes «à double auxiliation » à une série de tests d'inaccusativité, Lagae (2005 : 134) affirme qu'ils «partagent manifestement plus de propriétés avec les verbes inaccusatifs qu'avec les verbes inergatifs ». Leur principal argument serait donc interne au groupe verbal :

$$
\begin{aligned}
& {[\mathrm{P} \varnothing[\mathrm{sv} \text { V SN]] Verbes intransitifs à double auxiliation }} \\
& \mathrm{ex}: \text { Pierre est divorcé. }
\end{aligned}
$$

Sur le plan sémantique, les verbes aboutir, atterrir, disparaître, divorcer, expirer, paraître marquent l'aspect télique. Leur sujet correspond à un thème, c'est-à-dire à l'entité se trouvant dans un certain état ou affectée par l'action du verbe (subissant un changement d'état ou de lieu). Nous concluons donc que les verbes essentiellement intransitifs permettant la double auxiliation en avoir ou être suivi du participe passé sont également des verbes inaccusatifs, dont la structure argumentale se présente comme :

Pierre a divorce / Pierre est divorcé.

$((\mathrm{x}))$

Thème

\subsection{Les verbes à renversement ${ }^{4}$ et la forme « être + participe passé »}

\subsection{1 "être + participe passé » : un marqueur de structure argumentale}

La forme en «être + participe passé » issue de verbes à renversement comme couler, casser, geler, etc., peut être appréhendée en termes de structure argumentale. En effet, il est connu que les verbes à renversement entrent au moins dans deux constructions différentes : l'une transitive de type $S N_{1} V S N_{2}$, où $S N_{l}$ est agentif et $S N_{2}$ porte le rôle sémantique de thème, l'autre intransitive de type $S N_{2} V$ où $S N_{2}$ porte le rôle de thème. L'argument occupant la place du sujet dans la structure intransitive présente donc des 
caractéristiques d'un objet. Il a été avancé que les énoncés du type $S N_{2} V$ résulteraient d'une suppression de l'argument externe $S N_{1}$ de sorte que la position préverbale se libère et peut accueillir l'argument interne $S N_{2}$ (Lamiroy 1993, Burzio 1986) :

$$
[\mathrm{p} \varnothing[\mathrm{sv} \mathrm{V} \mathrm{SN}]] \quad \text { Verbes à renversement }
$$

Dans leur emploi intransitif et donc quel que soit l'auxiliaire sélectionné, ces verbes répondent donc aux critères définitoires de la classe des inaccusatifs et présentent la structure argumentale suivante (Burzio 1986 ; Zribi-Hertz 1987 ; Grimshaw 1992)

La branche est cassée/ La branche a cassé.

$(\mathrm{x}-\varnothing \quad(\mathrm{y}))$

Agent Thème

\subsection{2 « être + participe passé » : un marqueur d'aspect ?}

La séquence «être + participe passé » formée à partir d'un verbe à renversement présente une ambiguïté structurelle. L'exemple (24b) montre que cette forme peut, à l'instar des verbes à double auxiliation, être analysée comme exprimant l'état résultant de l'événement antérieur (24a). Une analyse comme forme passive est toutefois également possible et la forme «être + participe passé » manifestera à ce titre la même ambivalence aspectuelle (24d) que le passif que nous aborderons dans le paragraphe suivant.
a. Son navire a coulé.
b. Son navire est coulé, sa vie est révolue. (A. de Vigny, Les Destinées)
c. L'artillerie a coulé son navire.
d. Regarde! Son navire est coulé. ('est en train d'être coulé' / 'est au fond de l'eau')

Quoique la forme «être + participe passé » marquant l'aspect résultatif existe pour la plupart de verbes à renversement, elle fait défaut pour des verbes comme bleuir, sécher, grossir, mûrir étant donné la concurrence d'un adjectif qui leur est morphologiquement apparenté (Zribi-Hertz 1987 ; Creissels 2002 ; Lagae 2005).

$$
\text { *Le linge est séché depuis longtemps / le linge est sec depuis longtemps. }
$$

\subsection{Le passif périphrastique " être + participe passé »}

\subsection{1 "être + participe passé » : un marqueur de structure argumentale}

Selon Desclés \& Guentchéva (1985), Grimshaw (1992) et Lamiroy (1993), la fonction du passif consiste à dégrader l'argument externe du verbe en le supprimant ou en lui assignant la fonction de circonstant. En ce sens, la construction passive correspond fondamentalement à une structure inaccusative : l'argument unique correspond à l'argument interne direct.

(26) $\quad[\mathrm{p} \varnothing[\mathrm{sv}$ V SN] $] \quad$ Structure passive

Du point de vue de sémantique, cet argument interne porte le rôle de thème.

Le décor est installé.

$(\mathrm{x}-\varnothing \quad(\mathrm{y}))$

\section{Agent Thème}

Contrairement aux cas étudiés précédemment, «être + participe passé » formé à partir d'un verbe transitif marque le caractère inaccusatif de la construction verbale et non pas du verbe en tant que lexème. 


\subsection{2 « être + participe passé » : un marqueur d'aspect ?}

Si le passif périphrastique peut être abordé en termes de structure argumentale, il a également fait l'objet d'une analyse aspectuelle. En effet, il a été observé que les phrases passives issues de procès téliques sont ambiguës sur le plan aspectuel (Meigret 1550; Authier 1972; Blanche-Benveniste 1984; Vikner 1985 ; Skårup 1998 ; Blanche-Benveniste 2000 ; Creissels 2000 et Carlier 2002). Ainsi, la phrase passive (28a), plutôt que d'être en relation de paraphrase avec (28b), tend à marquer l'état résultant de l'événement antérieur (28c) :
a. Une petite lampe est posée par terre. (P. Claudel, Tête d'or)
b. On pose une petite lampe par terre.
c. On a posé une petite lampe par terre.

Carlier (2002) fait remarquer que l'insertion d'un verbe processif non télique dans une structure passive peut également engendrer un glissement vers un sens statif : l'insertion de la locution être en train de, toujours compatible avec les verbes processifs non téliques à l'actif, est parfois peu naturelle voire exclue avec ces mêmes verbes au passif. Aussi être surveillé dans (29a) peut-il être glosé comme 'être sous surveillance'.

(29) a. Marie est en train de surveiller les enfants.

b. ??Les enfants sont en train d'être surveillés par Marie.

\subsection{La construction pronominale et la forme « être + participe passé »}

\subsection{1 "être + participe passé » : un marqueur de structure argumentale}

Plusieurs études ont analysé de façon unitaire les constructions pronominales du point de vue de la structure argumentale. Ainsi, Melis (1990: 121-126) et Grimshaw (1992: 152-158) montrent que les sujets des tours pronominaux peuvent être analysés comme des arguments internes. Les deux auteurs soutiennent ainsi que le syntagme nominal occupant la position de sujet, coréférentiel au clitique réflexif, correspond toujours à l'argument interne au groupe verbal. Pour Melis, le pronom réflexif se doit être analysé comme un accusatif qui ne réalise pas de position d'argument. La corrélation entre l'objet et la position d'argument interne étant d'abord éprouvée, l'argument interne ne pouvant pas recevoir le cas accusatif monte en position sujet. Comme se réfère au sujet, le syntagme nominal monté en position sujet sera considéré comme réalisation possible de l'argument interne. Deux cas de figure peuvent alors être envisagés :

1) L'argument interne répond également aux contraintes qu'impose le verbe à l'argument externe et il cumule alors les deux positions. On aura alors un tour agentif.

Tours subjectifs ex : Pierre se rase

$$
\begin{array}{ll}
(\mathrm{x}=\mathrm{y} \quad(\mathrm{y}) & ) \\
\text { Agent Thème }
\end{array}
$$

2) L'argument interne ne répond pas aux contraintes qu'impose le verbe à l'argument externe. Il est alors « traité comme une variable non spécifiée ». On a alors un tour non agentif.

$$
\begin{array}{r}
\text { Tours objectifs ex : la branche se casse } \\
(\mathrm{x}-\varnothing \quad(\mathrm{y}))
\end{array}
$$

Agent Thème

Cette analyse fait donc des constructions pronominales des structures fondamentalement inaccusatives. 


\subsubsection{La structure « être + participe passé » : un marqueur d'aspect ?}

A l'inverse des constructions pronominales non téliques (32), une grande partie des constructions pronominales à valeur aspectuelle télique admettent la forme "être + participe passé » à valeur d'état résultant, qu'il s'agisse de tours objectifs (33) ou de tours subjectifs (34) (Zribi-Hertz 1987 ; Creissels 2000 ; Lagae 2005).

a. Pierre s'est promené.

b. ?Pierre est promené.

a. Le mur s'est écroulé.

b. [...] ils doivent se rejoindre à l'endroit où le mur est écroulé. (C. Simon, L'Herbe)

a. Pierre s'est rasé.

b. L'homme est habillé en civil, il est rasé, il a l'air de beaucoup souffrir. (M. Duras, La Douleur)

Dans le cas de «être + participe passé » formé à partir d'un tour subjectif, le sujet porte exclusivement le rôle de thème et non pas celui d'agent.

Tout comme dans le cas des verbes à renversement, la forme résultative en «être + participe passé » peut faire défaut quand le participe passé est concurrencé par un adjectif qui lui est morphologiquement apparenté :
a. Le temps s'est amélioré
b. *Le temps est amélioré.
c. Le temps est meilleur.

\section{Le participe passé des séquences en " être + participe passé » : de la sphère adjectivale à la sphère verbale}

\subsection{Introduction}

Dans cette partie, nous nous pencherons sur le statut catégoriel du participe passé dans les différentes séquences de «être + participe passé » distingués précédemment et nous préciserons ses rapports avec l'adjectif et le verbe. Afin de tester le statut adjectival des participes passés, nous les modifierons tout d'abord par l'adverbe très (Riegel 1985 et Goes 1999). Il est en effet connu que très se rapporte à des adjectifs susceptibles de gradation (36a) mais est exclu avec les verbes présentant la même propriété (37).
a. L'homme est grand, ses yeux sont très bleus, ses lèvres épaisses. (D. Belloc, Kepas)
a. *Il lit très le soir.

Il est ainsi en distribution complémentaire avec beaucoup, pouvant modifier des verbes mais exclu avec les adjectifs.

b. *Ses yeux sont beaucoup bleus.

(36) b. Donc, il lit beaucoup le soir ? (P. Sollers, Le Secret) 
Nous remplacerons ensuite être par d'autres verbes copules, ce remplacement étant possible dans la structure «être + adjectif» sous réserve de variations modales ou aspectuelles (Riegel 1985).
a. Le mur est blanc.
b. Le mur semble / paraît / a l'air blanc (sur cette photo).

Enfin, nous examinerons si les participes passés permettent à l'instar des adjectifs la substitution par le pronom invariable le (Riegel 1985 ; Le Goffic 1993 ; Goes 1999 ; Noailly 1999).

$$
\text { Innocent ... personne ne l'est. (J.-B. Pouy, La Clef des mensonges) }
$$

Afin de tester le statut verbal des participes passés, nous les modifierons par bien, qui dans son sens qualitatif et en tant qu'antonyme de mal, ne peut se rapporter aux adjectifs. Nous examinerons dans la même perspective la compatibilité avec l'adverbe mieux (Rivière 1990).

a. Il faut mieux décorer / éclairer cette pièce.

b. Cette pièce est mieux *petite / *claire.

(41) Il écrit bien / mal.

(42) Elle est bien gentille / *mal gentille.

Le deuxième test que nous utilisons pour mettre en évidence la nature verbale de «être + participe passé » est son aptitude à entrer dans une structure impersonnelle (Rivière $1981: 49)$.

(43) Il entre un homme.

(44) *Il est heureux un homme.

\subsection{Les verbes intransitifs ayant un passé composé en «être + participe passé ॥}

L'ensemble des tests mettent en évidence la nature verbale du participe passé entrant dans la composition du passé composé quand celui-ci à une valeur d'antériorité ${ }^{5}$. Ainsi être ne peut jamais commuter avec paraître, sembler ou avoir l'air pour former le même temps verbal, le participe passe ne peut être cliticisé par le et la forme «être + participe passé » entre sans difficulté dans une construction impersonnelle.

(45) *Il paraît parti la semaine dernière.

(46) Pierre est arrivé précipitamment. / *Pierre l'est précipitamment.

(47) J'avais déjà commencé à manger lorsqu'il est entré une bizarre petite femme qui m'a demandé si elle pouvait s'asseoir à ma table. (A. Camus, L'Etranger) 
Quand le passé composé marque le présent d'aspect accompli à valeur résultative, les faits sont moins nets. La commutation de être avec d'autres verbes copules est toujours possible avec le participe mort et n'est pas exclue avec certains autres verbes. De même, la clitisation des participes passés par le n'apparaît pas exclue. Aussi le rapprochement avec la structure «être + adjectif attribut » semble possible.

(48) Là, près des grosses branches de l'arbre qui a l'air mort. (G. Duhamel, Le Désert de Bièvres)

(49) Elle est dehors, toute seule sur le perron, où elle accueillait les invités. Mais tout le monde paraît arrivé à cette heure, et la belle fiancée s'attarde inutilement. (M. Tournier, Les Météores)

(50) [...] présentant dans leurs récits comme arrivés des événements qui ne le sont pas encore. (E. Mounier, Traité du caractère)

Toutefois, la nature verbale du passé composé à valeur d'accompli ressort du fait que la structure impersonnelle est possible.

(51) ... on prend le même parcours, la crevasse, elle glisse... il est tombé un peu de neige... pas beaucoup, une poudre... en avant donc pour ce train, nos plates-formes! (L.-F. Céline, Rigodon)

«Etre + participe passé » est donc fondamentalement une structure verbale quand elle correspond à un passé composé antérieur et présente à la fois des propriétés verbales et des propriétés d'une structure attributive adjectivale quand il s'agit d'un passé composé à valeur accomplie.

\subsection{Les verbes intransitifs dits « à double auxiliation »}

Les participes passés des formes résultatives issues de verbes intransitifs à double auxiliation présentent majoritairement des caractéristiques adjectivales ${ }^{6}$. Ainsi, la plupart des formes permettent le remplacement de être par un autre verbe copule, acceptent la pronominalisation du participe passé par le et sont exclues de la structure impersonnelle :

(52) Ce malheureux traité du 15 juillet a presque détruit en un jour l'ouvrage de dix années et il a fait revivre les souvenirs, les animosités et surtout les défiances qui semblaient disparus. (A. de Tocqueville, Correspondance avec H. Reeve)

(53) [...] la quasi-totalité des annonces parues le sont sur les rares sites spécialisés dans ce domaine, tandis que les sites généralistes ignorent superbement les métiers et compétences liés aux sciences de l'information. (www.cepid.com)

(54) *Il est divorcé beaucoup de couples.

Néanmoins, les formes en «être + participe passé » correspondant aux verbes divorcer et naufrager ne permettent pas, quant à elles, qu'au verbe être se substituent d'autres verbes copules et elles se distinguent sur ce point des structures «être + adjectif ».

?Cet homme semble divorcé.

(56) ?Les personnages semblent naufragés.

Comme la majorité des verbes répondent positivement à ce test, on admettra que «être + participe passé » se présente d'une manière dominante comme une structure attributive adjectivale quand cette forme correspond à l'état résultant d'un verbe dit à double auxiliation. 


\subsection{Les verbes à renversement ayant un état résultant en " être + participe passé ॥}

Les formes en «être + participe passé » issues des verbes à renversement comme caraméliser, casser, geler, cicatriser, cuire, gonfler, etc., tendent à se rapprocher des structures en «être + adjectif». En premier lieu, elles sont susceptibles d'être modifiées par très et s'opposent sur ce point aux formes correspondantes «avoir + participe passé », où la modification d'intensité doit se faire au moyen de beaucoup. En second lieu, à être peut être substitué un verbe copule. Troisièmement, elles permettent la clitisation du participe passé par le. Enfin, elles n'entrent pas dans la structure impersonnelle ${ }^{7}$.

(57) a. Mais même si un contact est rapidement établi avec la sonde, "nous ne serons peut-être pas capables de creuser sur $50 \mathrm{~cm}$ si le sol est très gelé. (exobio.chez-alice.fr) / 'Le sol est beaucoup gelé.

b. Le sol a beaucoup gelé. / *Le sol a très gelé.

(59) Ici, bon nombre de fenêtres sont encore entières, ce qui est rare. Celles qui sont cassées le sont souvent à cause du vent. (tchorski.morkitu.org)

(60) *Il est gelé un lac.

Toutefois, certaines de ces formes acceptent la modification par bien / mal / mieux, ce qui met en évidence que les participes passés ont des propriétés verbales.

(61) Si la plaie est mal cicatrisée, le médecin peut décider d'attendre jusqu'à un an et plus avant de reprendre la cicatrice vicieuse. (www.e-sante.fr)

«Etre + participe passé » formé à partir d'un verbe de renversement se rapproche d'une structure attributive adjectivale quand elle correspond à l'état résultant d'un verbe à renversement. La modification du participe passé par mieux / bien empêche toutefois de l'assimiler à un simple adjectif.

\subsection{Le passif périphrastique " être + participe passé »}

La plupart des tests font ressortir une distinction entre les passifs à valeur processive et les passifs à valeur stative. Les passifs à valeur processive présentent plusieurs caractéristiques verbales : ils refusent le remplacement de être par un autre verbe copule, sont modifiés par mieux / bien et peuvent sans difficulté être utilisés dans une structure impersonnelle.

*La maison semble / paraît construite en ce moment.

(63) Les peaux tannées tombèrent dans l'eau et furent mal lavées par les soldats français dans les eaux de la Sambre à hauteur du gué de Floreffe. (www.tanneurs.com)

(64) En 1854 il est construit une école normale d'institutrices animée par la congrégation des soeurs de Nevers. (www.emsam.terre.defense.gouv.fr)

Le participe qui entre dans sa composition se rapproche toutefois de l'adjectif en acceptant la pronominalisation par le et révèle sa double nature, verbale et adjectivale, en acceptant la modification tant par très que par beaucoup.

(65) La première église fut construite en 1742; la seconde le fut en 1790. (www.uquebec.ca)

(66) Trois maisons furent très endommagées [...]. (www.azurseisme.com)

(67) Les maisons ont été beaucoup endommagées. 
Les passifs à valeur stative manifestent davantage de caractéristiques adjectivales : ils sont susceptibles d'être modifiés par très, ils permettent que se substituent à être d'autres verbes copules et ils acceptent la pronominalisation du participe passé par le.

(68) J'imagine, je fais l'itinéraire, je calcule le temps dont je dispose, je suis très surveillée par ma mère. (A. Ernaux, La femme gelée)

(69) Un ouvrage d'une gaieté infernale qui semble écrit par un être d'une autre nature que nous [...]. (P. Sollers, Le Secret)

(70) Oui, sa carte est tachée et mes gants le sont aussi. (E. About, Le Nez d'un notaire)

Leur nature verbale apparaît toutefois par le fait qu'ils peuvent également être modifiés par beaucoup comme par mieux / bien et qu'ils entrent dans la structure impersonnelle :

(71) Or, le meilleur de tous les calculs, c'est d'avoir des pièces qui soient beaucoup jouées, dont le succès constaté fasse marcher la province [...]. (G. Sand, Correspondance)

(72) La voiture est mal garée. (M. Embareck, Sur la ligne blanche)

(73) Sur chaque face, il est enregistré trois chansons. (Hériau 1980)

Quoique les tests tendent à montrer que «être + participe passé » correspondant à un passif processif soit de nature verbale, ils mettent surtout en exergue la double nature du passif - à la fois verbe et adjectif quelle que soit sa valeur. Ces observations invalident l'hypothèse de Damourette \& Pichon (1911-40) et de Helland (2000), postulant l'existence de deux structures différentes, une structure proprement passive «être + participe passé » pour la valeur processive et une structure «être + adjectif» pour la valeur stative.

\subsection{Les constructions pronominales ayant un état résultant en «être + participe passé ॥}

Des tests se dégage que «être + participe passé » formé à partir des constructions pronominales présente des caractéristiques d'une structure attributive adjectivale. Ainsi, alors que la forme pronominale, processive, n'accepte que la modification au moyen de l'adverbe beaucoup et est incompatible avec très, la forme «être + participe passé », qui en exprime l'état résultant, est fréquemment combinée avec très (74). De plus, cette forme à valeur d'état résultant permet le remplacement de être par d'autres verbes copules (75), elle accepte la pronominalisation du participe passé par le (76) et elle refuse la construction impersonnelle (77).

(74) a. La cigale d'Anouilh représente une personne élégante : elle est très maquillée et bien habillée : "l'œil noyé sous le fard », " un regard d'acier briller », [...]. (www.php.acorleans-tours.fr) / *Elle est beaucoup maquillée et bien habillée.

b. Elle s'est beaucoup maquillée. /* Elle s'est très maquillée.

(75) Louis Laine reste couché par terre, les yeux fermés. (P. Claudel, L'Echange)

(76) Je suis documenté, et vous ne l'êtes point. (Alain, Propos)

(77) ?Il est écroulé un mur.

Néanmoins, la modification par mieux / bien met en évidence que ces formes présentent par ailleurs aussi des caractéristiques verbales (78).

(78) Vous êtes bien assis? Non, n'est-ce pas? Vague mépris, même, hein? (D. Pennac, La Petite Marchande de prose) 
«Etre + participe passé » correspondant à l'état résultant d'une construction pronominale tend ainsi à se rapprocher de la structure «être + adjectif», sans pourtant s'y assimiler, contrairement à ce qui est affirmé par Skårup (1998 : 262-263) et Lapierre (2001 : 92 et 107).

\subsection{Bilan}

Le tableau suivant relève, pour chacune des différentes instances de la forme «être + participe passé » passées en revue, les propriétés verbales et les propriétés adjectivales du participe passé. Sont également indiqués, par les cases hachurées, les traits qui n'ont pas pu être testés.

\begin{tabular}{|c|c|c|c|c|c|c|c|c|c|}
\hline & Verbe & $\begin{array}{c}\text { PC } \\
\text { Antér. }\end{array}$ & $\begin{array}{c}\text { Passif } \\
\text { Processif }\end{array}$ & $\begin{array}{c}\text { PC } \\
\text { Accom- } \\
\text { pli }\end{array}$ & $\begin{array}{l}\text { Passif } \\
\text { Statif }\end{array}$ & $\begin{array}{c}\text { Etats } \\
\text { résultatifs } \\
\text { constr. } \\
\text { pronom }\end{array}$ & $\begin{array}{c}\text { Etats } \\
\text { résult. } \\
\text { verbes à } \\
\text { renv. }\end{array}$ & $\begin{array}{c}\text { Etats } \\
\text { résult. } \\
\mathbf{V} \\
\text { à double } \\
\text { aux. }\end{array}$ & $\begin{array}{l}\text { Adject } \\
\text { if }\end{array}$ \\
\hline $\begin{array}{c}\text { Très / } \\
\text { Beaucou }\end{array}$ & & & & & & & & & \\
\hline $\begin{array}{c}\text { Pronom } \\
\text { le }\end{array}$ & & & & & & & & & \\
\hline $\begin{array}{l}\text { Verbes } \\
\text { condoc }\end{array}$ & & & & & & & & & \\
\hline $\begin{array}{c}\mathrm{Bien} / \\
\mathrm{mal} / \mathrm{mie}\end{array}$ & & & & & & & & & \\
\hline $\begin{array}{l}\text { Imper- } \\
\text { sonnel }\end{array}$ & & & & & & & & & \\
\hline
\end{tabular}

D'après nos tests, le continuum suivant se dessine du verbe à la structure attributive faisant intervenir un adjectif :

Verbe Adjectif

$\mathrm{PC}$ antérieur > passif processif > PC accompli > passif statif > état résultant de V. à renv. / des constr. pronom > état résultant des V. à double auxiliation

Le participe passé auxilié par être ne peut donc jamais être totalement assimilé ni à la structure à attribut adjectival ni à une forme purement verbale. Sa proximité par rapport à la sphère adjectivale fait ressortir un degré moins avancé de grammaticalisation. Son intégration dans la flexion verbale atteste un stade plus avancé de grammaticalisation.

Le passif a parfois été distingué du passé composé par un rapprochement par rapport à la structure «être + attribut » (Abeillé \& Godard 2002). Les tests utilisés ici mettent en évidence qu'il convient de prendre en compte également l'opposition aspectuelle entre valeur processive et valeur stative.

\section{La genèse de la forme verbale " être + participe passé » en tant que marqueur de l'aspect et de la structure argumentale}

Le développement de «être + participe passé » en tant que forme fléchie du verbe et l'extension de ses conditions d'emploi doivent être envisagés dans le contexte global d'un changement typologique global qui caractérise l'évolution du latin au français : le latin, ayant des caractéristiques d'une langue OV, tend à marquer la grammaire sous forme de morphèmes suffixés à la tête, alors que le français, étant une langue VO, exprime la grammaire sous forme de morphèmes libres précédant la tête (Geisler 1982). C'est ainsi qu'au cours de l'évolution du latin au français, tant le parfait que le passif se voient dotés 
d'une forme faisant intervenir un auxiliaire suivi d'une forme verbale non finie. Reste la question de savoir pourquoi l'auxiliaire être a été adopté aussi bien pour le parfait que pour le passif.

\section{1 "esse + participe passé » en tant que marqueur de la structure argumentale}

Le participe passé a été formé par analogie avec l'adjectif et la structure « esse + participe passé » marque ainsi un état sur le modèle de la structure copulative à attribut adjectival. L'intégration de la séquence «esse + participe passé » dans la flexion verbale en tant que forme passive conduit à concevoir cet état comme l'aboutissement du procès exprimé par le verbe (Cohen 1989 : 111). Cette construction verbale est inaccusative : l'argument unique, qui est présenté comme se trouvant dans un état résultant du procès exprimé par le verbe, détient le rôle de thème par rapport à ce verbe. L'agent peut être facultativement exprimé sous forme d'adjoint.

Ce stade d'évolution est atteint en latin classique, qui utilise le présent du verbe esse combiné au participe passé pour exprimer le parfait passif et, d'une manière analogue, les formes de l'imparfait et du futur du verbe esse suivies du participe passé pour former le plus-que-parfait et le futur exact. Comme le note Menge (2000: § 141), on relève déjà parallèlement les formes du parfait, du plus-que-parfait du futur exact suivies du participe passé avec la même fonction :

$$
\begin{aligned}
& \text { Pons, qui fuerat tempestate interruptus, ... (Caesar, Commentarii belli civilis 1, 41, 1) } \\
& \text { 'Le pont, qui avait été interrompu par une tempête, ... }
\end{aligned}
$$

C'est dire que la forme du présent de esse combiné au participe passé n'est plus restreinte à l'expression de l'état résultant d'un procès antérieur, mais peut également déjà exprimer un procès en cours. C'est ainsi qu'on s'approche du stade suivant de l'intégration de la forme «être + participe passé » dans la flexion verbale. La forme «être + participe passé » peut perdre sa valeur stative pour devenir pleinement un marqueur de structure argumentale et il finira par remplacer dans ce nouveau rôle les anciennes formes synthétiques du passif présent, imparfait et futur simple. Cette intégration reste pourtant imparfaite : l'ambivalence du passif de la forme «être + participe passé » existe encore en français moderne. Ainsi, dans (31), le passif tend en effet à être interprété en tant qu'état résultant, sans que l'interprétation de procès en cours soit exclue hors contexte (Blanche-Benveniste \& al. 1984, Carlier 2002).

$$
\begin{aligned}
& \text { a. Une petite lampe est posée par terre. (P. Claudel, Tête d'or) } \\
& \text { b. On pose une petite lampe par terre. } \\
& \text { c. On a posé une petite lampe par terre. }
\end{aligned}
$$

Ce statut ambivalent du passif ressort dans le tableau : dans la mesure où le passif marque un procès, il se présente comme plus verbal, alors que le passif exprimant l'état résultant reste davantage ancré dans la structure à adjectif attribut qui constitue son origine. Par ailleurs, la valeur d'état résultant est la seule interprétation possible pour la forme "être + participe passé » si cette forme entre en concurrence avec une autre forme verbale dont la valeur aspectuelle est non stative, comme dans le cas de «être + participe passé » rattaché à un verbe à renversement ou à une construction pronominale. Aussi relève-t-on dans ces cas des propriétés nettement adjectivales.

\section{2 « esse + participe passé » en tant que marqueur du parfait et d'antériorité}

Le parfait étant également associé à un état résultant d'un procès antérieur, il a été exprimé dans diverses langues indo-européennes à un stade ancien de leur évolution au moyen de la forme verbale "être + participe parfait » (Benveniste 1966). Voici des traces de cette structure en latin.

(80) De reliquo antiqua fere formula utuntur. Cum emptor dixit :"tanti sunt mi [=mihi] emptae [oves]?" Et ille respondit : "sunt" (Varro, De Agri Cultura, cité par Benveniste 1966 : 204) ('Les ai-je acheté pour tant?') 
Cet état résultant est attribué à l'argument correspondant au thème du procès verbal et cette construction est donc également inaccusative. Comme le souligne Benveniste (1966:176-186), il y a pourtant une différence par rapport au passif : l'agent ou l'expérienceur est maintenu en tant qu'argument du verbe et s'exprime en latin sous la forme d'un datif.

C'est à partir de cette structure que se développe l'auxiliation avec habere ('avoir'). Comme le met en évidence l'étude bien connue de Benveniste (1966 : 187-207) intitulée « être et avoir dans leurs fonctions linguistiques », avoir est à analyser comme un être-à inversé : «Mihi est pecunia se retourne en habeo pecuniam» (1966: 197). C'est ainsi que la structure tanti sunt mihi emptas dans l'exemple (80) peut se transformer en tanti habeo emptas, où l'emploi de habere annule la structure inaccusative du parfait.

La structure qui combine habere avec le participe passé n'est pas encore grammaticalisée en latin, mais doit être analysée comme une structure à prédication seconde où le participe se rapporte à l'objet direct de habere à la manière d'un attribut. Pinkster (1987) et Jacob (1998) mettent en évidence les différentes étapes du développement qui conduiront finalement à la réanalyse de la séquence « habere + participe parfait » comme forme verbale complexe :

Chez les auteurs préclassiques, habere prend le plus souvent le sens de 'maintenir' quelqu'un ou quelque chose dans un état et habere confère ainsi à son sujet le rôle d'agent.

Inclusum in Curia senatum habuerunt (Cicéron, Att. 6,2,8)

('ils tinrent le sénat enfermé dans la Curie')

- Dès l'âge classique, habere se combine fréquemment avec des verbes de perception et le sujet de habere a ainsi le rôle d'experienceur.

Clodii animum perspectum habeo, cognitum, iudicatum (Cicéron, ad Brut, 1,1,1)

('j'ai depuis longtemps pénétré, sondé, jugé l'âme de Clodius. ...')

- En latin tardif se maintient surtout un troisième type d'emploi où habere attribue au sujet le rôle d'agent moralement responsable.

(84) Si habent etiam cum daemonibus initam societatem (Aug. Doctr christ 2, 39,58, cité par Pinkster 1987 : 203) ('ils ont même engagé une alliance avec des démons')

Il apparaît ainsi que dans ses premiers emplois, le verbe habere introduit une position argumentale en y attribuant le rôle d'agent, qui s'affaiblira par la suite. Cette évolution d'affaiblissement aboutit au moment où habere, devenu auxiliaire, perd entièrement sa structure argumentale et sa capacité de distribuer des rôles sémantiques, de sorte que la position argumentale et le rôle sémantique du sujet syntaxique de habere sont déterminés par le verbe au participe passé.

L'origine historique du parfait «habere + participe passé » permet de comprendre pourquoi les verbes inaccusatifs, dépourvus de position argumentale pour un agent, ont pu maintenir l'auxiliation au moyen du verbe esse. La progression de la grammaticalisation de la forme verbale «habere + participe passé » a néanmoins eu pour conséquence que cette forme s'est généralisée dans certaines langues pour l'expression du parfait en évinçant l'auxiliaire esse dans le domaine du parfait ${ }^{8}$. Tel est le cas de l'espagnol'. L'italien, au contraire, maintient intacte l'opposition entre les verbes et constructions verbales inaccusatives, auxiliés avec essere, et les verbes ou constructions verbales transitives ou inergatives, sélectionnant pour l'expression du parfait l'auxiliaire avere. Le français occupe une position intermédiaire, dans la mesure où le domaine d'emploi de l'auxiliaire être s'est rétréci au cours de l'histoire. A la différence de l'italien, le français moderne ne permet ainsi plus l'utilisation du verbe être dans le cas des verbes comme voler, courir, marcher accompagné d'un complément délimitant le procès.

(85) il estoit vollé desjà si loing, qu'elle ne le povoit plus rappeller (Marguerite de Navarre, L'Heptaméron (16 $6^{\mathrm{e}}$ siècle)) 
Le passé composé à valeur aspectuelle d'accompli de forme «être + participe passé » peut servir à la prédication d'un état résultant, mais peut également se dissocier de cette valeur stative liée à son origine pour évoquer un procès dynamique antérieur par rapport au moment de l'énonciation. Comme il ressort du tableau sous $\S 2.7$, dans cette dernière valeur, la forme «être + participe passé » ne dispose plus d'aucune propriété rappelant son origine dans la structure à attribut adjectif.

\section{Références bibliographiques}

Abeillé A. et Godard D., 2002, The syntactic structure of French auxiliaries, Language 78, 404-451.

Aranovich, R. (2003). The semantics of auxiliary selection in Old Spanish. Studies in Language 27.1: 1-37

Benveniste, E. (1966). Problèmes de linguistique générale I, Paris : Gallimard.

Blanche-Benveniste, C., Deulofeu J., Stéfanini J., Van den Eynde K. (1984), Pronom et syntaxe: l'approche pronominale et son application au français, Paris : SELAF.

Burzio, L. (1985). Italian Syntax. Dordrecht : Reidel.

Carlier, A. (2002). Les propriétés aspectuelles du passif. Cahiers Chronos, 10, 41-63.

Carlier, A. (2005). L'argument davidsonien : un critère de distinction entre les prédicats 'stage level' et 'individual level' ?. Travaux de Linguistique 50, 13-35.

Cohen, D. (1989). L'aspect verbal. Paris : PUF

Comrie B. (1981). Aspect and voice : some reflections on perfect and passive, in Syntax and semantics 14: 65-78.

Creissels, D. (2000). L'emploi résultatif de être + participe passé en français. Cahiers Chronos, 6, 133-142.

Cummins S. (2000), The unaccusative hypothesis and the impersonal construction in French, The Canadian journal of linguistics, 45, 225-251

Damourette, J. et Pichon, E. (1940-1944). Des mots à la pensée: essai de la grammaire de la langue française t. IV. Paris : d'Artrey.

Desclés, J.-P. et Guentchéva, Z. (1993). Le passif dans le système des voix du français. Langages, 109, 73-103.

Feuillet, J. (1984). Les fonctions du groupe adjectival. La linguistique, 20/2, 144-150.

Geisler, H. (1982). Studien zur typologischen Entwicklung: Lateinisch, Altfranzösisch, Neufranzösisch. München: Wilhelm Fink.

Goes, J. (1997). L'attribut: objet de être ? Travaux de linguistique, 34, 49-64.

Gosselin, L. (1996). Sémantique de la temporalité en français : un modèle calculatoire et cognitif du temps et de l'aspect. Louvain-la-Neuve : Duculot.

Grimshaw, J. (1992). Argument Structure. Cambridge: MIT Press.

Harris, M. (1987). The 'past simple' and the 'present perfect' in Romance, in Vincent N. \& Harris M (Eds). Studies in the Romance Verb. London: Croom Helm.

Helland, H.-P. (2000). Le passif verbal et le passif adjectival. Etudes Romanes, 45, 83-97.

Jacob, D. (1998). Transitivität, Diathese und Perfekt: zur Entstehung der romanischen haben-Periphrasen, in Geisler H. \& Jacob D. Eds, Transitivität und Diathese in romanischen Sprachen. Tübingen: Niemeyer.

Kuryłowicz, J. (1973). Les temps composés du roman. in Kuryłowicz J. Ed. Esquisses linguistiques I, München : Wilhelm Fink].

Lagae, V. (2005). Les formes en «être + participe passé » à valeur résultative dans le système verbal français », Cahiers Chronos, 12, 125-142. 
Lamiroy, B. (1993). Pourquoi il y a deux passifs. Langages, 109, 53-72.

Lamiroy, B. (1999). Auxiliaires, langues romanes et grammaticalisation. Langages, 135, 63 - 75.

Le Goffic, P. (1993). Grammaire de la phrase française. Paris : Hachette Education.

Legendre, G., Sorace, A. (2004). «Auxiliaires et intransitivité en français et dans les langues romanes », dans D. Godard (éd.) Les langues romanes. Paris, CNRS- Edition, 183-233.

Levin, B. et Rappaport, M. (1995). Unaccusativity. At the syntax-lexical semantics interface. Cambridge-London : MIT Press.

Luscher, J.-M. et Sthioul, B. (1996). Emplois et interprétations du Passé Composé. Cahiers de Linguistique française, $18,187-217$.

Maranzt, A. (1984). On the nature of grammatical relations. Cambridge, MIT Press.

Martin, R. (1970). La transformation impersonnelle. Revue de Linguistique Romane, 34, 377-394.

Meigret, L. (1550 [1980]). Le traité de la grammaire française, éd. par F. J. Hausmann, Tübingen : G. Narr.

Melis, L. (1990). La voie pronominale. Paris / Louvain-la-Neuve : Duculot.

Menge H. (2000). Lehrbuch der lateinische Syntax und Semantik, Darmstadt : Wissenschaftliche Buchgesellschaft.

Perlmutter, D. (1978). Impersonal passive and the unaccusative hypothesis. Proceedings of the Fourth Annual Meeting of the Berkeley Linguistics Society. The University of California at Berkeley.

Pinkster H. (1987) The srategy and chronology of the development of future and perfect tense auxiliaries in Latin. In Harris M. \& Ramat P. Eds, Historical Development of Auxiliaries. 193-223, Berlin: Mouton de Gruyter.

Riegel, M. (1985). L'adjectif attribut. Paris : PUF.

Rivière, N. (1990). Le participe passé est-il verbe ou adjectif? Travaux de Linguistique et de Philologie, 131-169.

Ruwet, N. (1972). Théorie syntaxique et syntaxe du français. Paris : Seuil.

Skårup, P. (1998). Les emplois de être + participe passé en français contemporain. In Bilger M., Van den Eynde, K. et Gadet G., éds, Analyse linguistique et approches de l'oral, Louvain : Peeters.

Tasmowski-De Ryck L.(1993). «Les verbes inergatifs et inaccusatifs en français» dans Verbum Romanicum : Festchrift für Maria Iliescu, 285-295.

Van de Velde, D. (1996). Le spectre nominal. Louvain: Peeters.

Vincent, N. (1987). The development of the auxiliaries habere and esse in Romance. in Vincent N. \& Harris M.(Eds) Studies in the Romance Verb. London: Croom Helm.

1 Deux verbes sélectionnant exclusivement être au passé composé, à savoir rester et provenir, ne se présentent pas du point de vue de leur aspect lexical comme des procès téliques, mais comme des états. Quoique rester doive être analysé comme un état transitoire, son sens lexical qui est 'continuer d'être dans un état ou un lieu', 'prolonger la durée de sa présence' fait que l'incidence induite par l'achèvement du procès se présente également comme un résultat
(1)
Comment
vous
appelez-vous
? demanda
Wolf.
- Je m'appelle Sandre, dit le grand marin, et mon copain se nomme Berzingue.
- Vous êtes restés dans le quartier depuis deux ans ? demanda Lazuli.
- Oui, dit Sandre. On y est bien. Nous aimons beaucoup le jeu. (B. Vian, L'Herbe rouge)

Quant à provenir, ayant pour sens en français contemporain 'avoir pour origine', il s'agit d'un état stable. Ce verbe n'est pas attesté au passé composé à valeur accomplie.

(2) *Ce vin est provenu d'Italie. 
${ }^{2}$ Cette hypothèse, originellement introduite par Perlmutter (1978) dans le cadre de la grammaire relationnelle, a été développée par Burzio (1986) à l'intérieur de la théorie du gouvernement et du liage. Les analyses spécifiques au français doivent beaucoup à Ruwet $(1972,1988)$. Certains auteurs comme Cummins (2000) remettent en question cette hypothèse tant du point de vue syntaxique que sémantique.

${ }^{3}$ Intervenir signifie entrer et prendre part à l'action dans un lieu concret (1) ou abstrait (2 et 3).

(1) Les soldats sont intervenus en Irak.

(2) Elle n'intervient pas dans l'éducation de ses enfants.

(3) Il est intervenu à ce colloque, dans cette discussion...

4 On trouve également les appellations «symétriques» ou « réversibles » pour désigner ces verbes. Une liste exhaustive en est fournie par Rothemberg (1974).

${ }^{5}$ Deux tests ne permettent pas de statuer quant à la nature verbale ou adjectivale des passés composés. Ainsi la plupart du temps, les verbes intransitifs ayant un passé composé en être sont incompatibles dans leur construction de base avec l'adverbe très ou beaucoup exprimant l'intensité tout comme avec les adverbes bien / mieux exprimant la qualité.

*Un incident advint mal / *Un incident est mal advenu.

Cette incompatibilité tient au fait que le procès verbal, qui dénote la venue à l'existence, la fin de l'existence ou la localisation, ne se prête ni à une gradation ni à une évaluation qualitative. Les passés composés correspondants ne peuvent donc être modifiés ni par très ou beaucoup à valeur d'intensité ni par mieux ou bien exprimant la qualité.

${ }^{6}$ La modification par très / beaucoup ou bien / mal / mieux n'est pas un test permettant de statuer sur le caractère verbal ou adjectival des verbes intransitifs formant leur accompli soit avec avoir soit avec être. En effet, la plupart des verbes intransitifs auxiliés soit par avoir soit par être sont incompatibles avec les adverbes dans la mesure ou ces verbes, qui dénotent comme les précédents la venue à l'existence, la fin de l'existence ou la localisation, ne se prêtent pas à une gradation à une évaluation par bien ou mieux.

(1) *Le dossier a mal disparu. / *Le dossier est mal disparu.

Seul le participe passé issu du procès aboutir peut être modifié par très et mieux mais il a alors le sens particulier de réussir.

(2) a. Il est vrai que ce CD, je l'ai acheté les yeux fermés, ou presque, en contradiction avec le titre de cet album qui ma foi est très abouti. (www.fnac.com)

(3) [...] franchement, cet OS [ = vista] est vraiment bon en tout cas, on peut dire qu'il est mieux abouti que son homologue XP [= windows XP). (www.revioo.com)

${ }^{7}$ L'incompatibilité entre les verbes à renversement et la structure impersonnelle nous semble être due au sémantisme de ces verbes, qui ne sont pas existentiels.

${ }^{8}$ Pour un aperçu de l'emploi des auxiliaires habere et esse dans les différentes langues romanes, voir Vincent (1987).

${ }^{9}$ L'extension de habere au détriment de esse au cours de l'histoire de l'espagnol a été étudiée par Aranovich (2003). 